\title{
Separate and joint similarity to families of normal operators
}

\author{
by \\ Piotr Niemiec (Kraków)
}

\begin{abstract}
Sets of bounded linear operators $\mathcal{S}, \mathcal{T} \subset \mathcal{B}(\mathcal{H})(\mathcal{H}$ is a Hilbert space) are similar if there exists an invertible (in $\mathcal{B}(\mathcal{H})$ ) operator $G$ such that $G^{-1} \cdot \mathcal{S} \cdot G=\mathcal{T}$. A bounded operator is scalar if it is similar to a normal operator. $\mathcal{S}$ is jointly scalar if there exists a set $\mathcal{N} \subset \mathcal{B}(\mathcal{H})$ of normal operators such that $\mathcal{S}$ and $\mathcal{N}$ are similar. $\mathcal{S}$ is separately scalar if all its elements are scalar. Some necessary and sufficient conditions for joint scalarity of a separately scalar abelian set of Hilbert space operators are presented (Theorems 3.7, 4.4 and 4.6).

Continuous algebra homomorphisms between the algebra of all complex-valued continuous functions on a compact Hausdorff space and the algebra of all bounded operators in a Hilbert space are studied.
\end{abstract}

In this paper we investigate the question of when a commutative family of (bounded linear) scalar operators on a Hilbert space (in the sense of Dunford and Schwartz) is (jointly) similar to a family of normal operators acting on the same space. Section 3 answers the question in case the family is arbitrary (see Theorem 3.7); the solution is written in terms of quasi-adjoints of the operators in question. In Section 4 we concentrate on families which are algebras. The main result (Theorem 4.4) says that any closed abelian algebra of scalar operators which is invariant under the operation of taking quasi-adjoints is automatically (jointly) similar to an abelian $\mathcal{C}^{*}$-algebra of normal operators. In particular, any closed abelian real algebra of operators similar to selfadjoint ones is (jointly) similar to the real part of an abelian $\mathcal{C}^{*}$-algebra of normal operators (Theorem 4.6).

Sections 1 and 2 deal with a single scalar operator. The literature concerning similarity to a normal operator is still growing and we mention just a few examples: Dunford and Schwartz [DS3], Sz.-Nagy [SzN], Gokhberg and Kreŭn [G-K], van Casteren [Ca1, Ca2], Naboko [Nab], Benamara and Nikolski [B-N].

2000 Mathematics Subject Classification: 47B40, 47B15. 
1. Preliminaries. In this paper $\mathcal{H}$ denotes a complex Hilbert space and $\mathcal{B}(\mathcal{H})$ the algebra of all bounded linear operators in $\mathcal{H}$. We denote by $\mathcal{G}(\mathcal{H})$ the multiplicative group of all invertible elements of $\mathcal{B}(\mathcal{H})$ and by $\mathcal{G}_{+}(\mathcal{H})$ the subset of $\mathcal{G}(\mathcal{H})$ consisting of all strictly positive (i.e. nonnegative and invertible) operators. For any $A \in \mathcal{B}(\mathcal{H})$ we denote by $\sigma(A)$ the spectrum of $A$ in $\mathcal{B}(\mathcal{H})$.

We follow the terminology of Dunford and Schwartz [DS3].

1.1. Definition. Let $\Omega$ be a nonempty set and $\mathfrak{M}$ be a $\sigma$-algebra of subsets of $\Omega$. A mapping $E: \mathfrak{M} \rightarrow \mathcal{B}(\mathcal{H})$ is called a spectral measure if:

(1) $E$ is an operator measure,

(2) $E(\sigma \cap \tau)=E(\sigma) E(\tau)$ for all $\sigma, \tau \in \mathfrak{M}$,

(3) $E(\Omega)=I_{\mathcal{H}}$.

If the mapping $E$ satisfies conditions (1)-(3) and all its values are nonnegative operators, then $E$ is said to be a nonnegative spectral measure.

Dunford and Schwartz [DS3] defined a scalar operator as an operator $A \in$ $\mathcal{B}(\mathcal{H})$ for which there exists a spectral measure $E: \mathfrak{B}(\sigma(A)) \rightarrow \mathcal{B}(\mathcal{H})$ such that $A=\int_{\sigma(A)} z d E(z)$. One may easily check that each bounded operator similar to a normal one is scalar (in the sense of Dunford and Schwartz). It turns out that the converse is also true - each scalar operator is similar to a normal one (see [DS3, Theorem XV.6.4]). Therefore we can state the following definition.

1.2. Definition. We say that a bounded operator is scalar if it is similar to a normal one.

1.3. Theorem ([DS3, Lemma XV.6.1]). Let $\mathcal{F} \subset \mathcal{G}(\mathcal{H})$ be any abelian subgroup of $\mathcal{G}(\mathcal{H})$ which is bounded, i.e. $\sup _{F \in \mathcal{F}}\|F\|<\infty$. Then there exists $G \in \mathcal{G}_{+}(\mathcal{H})$ such that for any $F \in \mathcal{F}$ the operator $G^{-1} F G$ is unitary.

A particular case of the above theorem is the classic Sz.-Nagy theorem which states that a bounded operator is similar to a unitary one if and only if it is invertible and the set of all its integer powers is bounded.

The following theorem is also a consequence of Theorem 1.3. It can be proved by the same argument as in the proof of [DS3, Lemma XV.6.2] $\left(^{1}\right)$.

1.4. TheOrem. Let $\mathcal{P} \subset \mathcal{B}(\mathcal{H})$ be any abelian (nonempty) set of projections (i.e. idempotents) such that

$$
P+Q-2 P Q \in \mathcal{P}, \quad P, Q \in \mathcal{P} .
$$

Then the following conditions are equivalent:

$\left({ }^{1}\right)$ Apply Theorem 1.3 to the abelian group $\{I-2 P: P \in \mathcal{P}\}$, where $\mathcal{P}$ is as in Theorem 1.4. 
(i) there exists $G \in \mathcal{G}(\mathcal{H})$ such that $G^{-1} P G$ is an orthogonal projection for every $P \in \mathcal{P}$,

(ii) $\sup _{P \in \mathcal{P}}\|P\|<\infty$.

Applying Theorem 1.4 to the range of a spectral measure, and the well known theorem which states that each spectral measure is bounded (see e.g. [DS1, Corollary IV.10.2]), we get

1.5. THEOREM (Dixmier). Let $\Omega$ be a nonempty set and $\mathfrak{M}$ be a $\sigma$-algebra of subsets of $\Omega$. Let $E: \mathfrak{M} \rightarrow \mathcal{B}(\mathcal{H})$ be a spectral measure. Then there exist a nonnegative spectral measure $E_{0}: \mathfrak{M} \rightarrow \mathcal{B}(\mathcal{H})$ and an operator $G \in \mathcal{G}_{+}(\mathcal{H})$ such that

$$
G^{-1} E(\tau) G=E_{0}(\tau), \quad \tau \in \mathfrak{M} .
$$

The following theorem is a starting point for our investigations.

1.6. Theorem ([DS3, Theorem XV.6.4 and Corollary XV.6.5]). If $A_{1}$, $\ldots, A_{n} \in \mathcal{B}(\mathcal{H})(n \geq 2)$ are commuting scalar operators, then there exists $G \in \mathcal{G}_{+}(\mathcal{H})$ such that $G^{-1} A_{j} G$ is normal for every $j \in\{1, \ldots, n\}$. Moreover, the operators $A_{j}+A_{k}$ and $A_{j} A_{k}$ are scalar $(j, k=1, \ldots, n)$.

The following well known fact is a consequence of Theorem 1.4 (for terminology see [Day, §3-4]).

1.7. Corollary ([Lor]). Let $\mathcal{H}$ be a separable (infinite-dimensional complex) Hilbert space and $\left(e_{n}\right)_{n=1}^{\infty}$ be an orthonormal basis of $\mathcal{H}$. Let $\left(f_{n}\right)_{n=1}^{\infty}$ be an unconditional Schauder basis of $\mathcal{H}$ such that $\sup _{n>1}\left\|f_{n}\right\|<\infty$ and $\inf _{n \geq 1}\left\|f_{n}\right\|>0$. Then there exists $G \in \mathcal{G}(\mathcal{H})$ such that $G\left(e_{n}\right)=f_{n}$ $(n \geq 1)$.

Proof. It follows from [Day, §3, Th. 1] that there exists a sequence $\left(l_{n}\right)_{n=1}^{\infty} \subset \mathcal{H}$ such that

$$
x=\sum_{n=1}^{\infty}\left\langle x, l_{n}\right\rangle f_{n}, \quad x \in \mathcal{H} .
$$

Denote by $P_{n}$ the mapping

$$
P_{n}: \mathcal{H} \ni x \mapsto\left\langle x, l_{n}\right\rangle f_{n} \in \mathcal{H}, \quad n \geq 1 .
$$

Then $\left(P_{n}\right)_{n=1}^{\infty} \subset \mathcal{B}(\mathcal{H})$ and $P_{j} P_{k}=\delta_{j k} P_{j}(j, k \geq 1)\left(^{2}\right)$. Set

$$
\mathcal{P}:=\left\{a_{1} P_{1}+\ldots+a_{n} P_{n}: n \geq 1, a_{1}, \ldots, a_{n} \in\{0,1\}\right\} .
$$

It is easy to check that $\mathcal{P}$ is an abelian set of projections which satisfies the condition: $P+Q-2 P Q \in \mathcal{P}$ for $P, Q \in \mathcal{P}$. Since the basis $\left(f_{n}\right)_{n=1}^{\infty}$ is unconditional, $\mathcal{P}$ is bounded (see [Day, §4, Theorem 1]). By Theorem 1.4 there exists $L \in \mathcal{G}(\mathcal{H})$ such that $L^{-1} P L$ is an orthogonal projection for any $P \in \mathcal{P}$. Let $\widetilde{f}_{n}:=L^{-1}\left(f_{n}\right)$ and $\widetilde{P}_{n}:=L^{-1} P_{n} L(n \geq 1)$. Then $\widetilde{P}_{n}\left(\widetilde{f}_{n}\right)=\widetilde{f}_{n}$,

$\left(^{2}\right) \delta_{j k}$ denotes the Kronecker delta. 
$\widetilde{P}_{n} \widetilde{P}_{m}=0(n, m \geq 1, n \neq m)$. This means that $\left(\widetilde{f}_{n}\right)_{n=1}^{\infty}$ is an orthogonal basis of $\mathcal{H}$. Moreover, $\sup _{n \geq 1}\left\|\widetilde{f}_{n}\right\|<\infty$ and $\inf _{n \geq 1}\left\|\widetilde{f}_{n}\right\|>0$. Set $\widetilde{e}_{n}:=$ $\widetilde{f}_{n} /\left\|\widetilde{f}_{n}\right\|(n \geq 1)$. Then $\left(\widetilde{e}_{n}\right)_{n=1}^{\infty}$ is an orthonormal basis of $\mathcal{H}$. Let $S \in \mathcal{B}(\mathcal{H})$ be defined by $S\left(\widetilde{e}_{n}\right):=\left\|\widetilde{f}_{n}\right\| \widetilde{e}_{n}=\widetilde{f}_{n}(n \geq 1)$. Then $S \in \mathcal{G}(\mathcal{H})$ (because $\inf _{n \geq 1}\left\|\widetilde{f}_{n}\right\|>0$ and $\left.\sup _{n>1}\left\|\widetilde{f}_{n}\right\|<\infty\right)$. We define a unitary operator $U$ by $U\left(e_{n}\right)=\widetilde{e}_{n}(n \geq 1)$. Finally, we put $G:=L S U \in \mathcal{G}(\mathcal{H})$. Now it is easy to check that $G\left(e_{n}\right)=f_{n}(n \geq 1)$.

It is worthwhile to mention here that there exists a Schauder basis in $l_{2}$ which is not unconditional (see [Bab]).

1.8. Lemma. Let $A$ be a scalar operator and $G_{1}, G_{2} \in \mathcal{G}(\mathcal{H})$ be such that $G_{1}^{-1} A G_{1}$ and $G_{2}^{-1} A G_{2}$ are normal. Then

$$
G_{1}\left(G_{1}^{-1} A G_{1}\right)^{*} G_{1}^{-1}=G_{2}\left(G_{2}^{-1} A G_{2}\right)^{*} G_{2}^{-1} .
$$

Proof. Set $M:=G_{1}^{-1} A G_{1}$ and $N:=G_{2}^{-1} A G_{2}$. By assumption, $M$ and $N$ are normal and $G_{1} M G_{1}^{-1}=A=G_{2} N G_{2}^{-1}$, which gives $\left(G_{2}^{-1} G_{1}\right) M=$ $N\left(G_{2}^{-1} G_{1}\right)$. By Putnam's theorem we get $\left(G_{2}^{-1} G_{1}\right) M^{*}=N^{*}\left(G_{2}^{-1} G_{1}\right)$, so $G_{1} M^{*} G_{1}^{-1}=G_{2} N^{*} G_{2}^{-1}$.

Now we can define the main notion of this paper.

1.9. Definition. Let $A$ be a scalar operator and $G \in \mathcal{G}(\mathcal{H})$ be such that $G^{-1} A G$ is normal. We define the quasi-adjoint of $A$ via

$$
A^{(*)}:=G\left(G^{-1} A G\right)^{*} G^{-1} .
$$

By Lemma 1.8 the quasi-adjoint is well defined, i.e. it does not depend on the choice of the operator $G$. In particular, the quasi-adjoint and the adjoint of a normal operator coincide.

Until the end of this section $\Omega$ denotes a compact (nonempty) Hausdorff space, $\mathcal{C}(\Omega)$ the algebra of all complex-valued continuous functions on $\Omega$, $\mathfrak{B}(\Omega)$ the $\sigma$-algebra of all Borel subsets of $\Omega$ and $\Phi: \mathcal{C}(\Omega) \rightarrow \mathcal{B}(\mathcal{H})$ an algebra homomorphism preserving units. $\mathcal{C}(\Omega, \mathbb{R})$ stands for the real algebra of all real-valued continuous functions on $\Omega$.

1.10. TheOREM ([DS3, Theorem XVII.2.5]). Let $\Phi: \mathcal{C}(\Omega) \rightarrow \mathcal{B}(\mathcal{H})$ be a continuous algebra homomorphism preserving units. Then there exists a spectral measure $E: \mathfrak{B}(\Omega) \rightarrow \mathcal{B}(\mathcal{H})$ such that

$$
\Phi(f)=\int_{\Omega} f d E, \quad f \in \mathcal{C}(\Omega) .
$$

Theorem 1.10 is also true for continuous algebra homomorphisms $\Phi$ : $\mathcal{C}(\Omega, \mathbb{R}) \rightarrow \mathcal{B}(\mathcal{H})$ preserving units.

The following theorem is well known and its proof is easy. 
1.11. TheOrem. Let $\mathcal{A}$ be a Banach algebra with unit and $\Phi: \mathcal{C}(\Omega) \rightarrow \mathcal{A}$ be a continuous algebra homomorphism preserving units. Then there exists a unique pair $\left(F, \Phi_{0}\right)$, where $F$ is a closed subset of $\Omega$ and $\Phi_{0}: \mathcal{C}(F) \rightarrow \mathcal{A}$ is a continuous algebra monomorphism preserving units, such that

$$
\Phi(f)=\Phi_{0}\left(\left.f\right|_{F}\right), \quad f \in \mathcal{C}(\Omega) .
$$

Moreover, $\operatorname{ker} \Phi=\left\{f \in \mathcal{C}(\Omega):\left.f\right|_{F} \equiv 0\right\}$.

1.12. Proposition. Let $\mathcal{A}$ be a (complex) Banach algebra with unit and $\Phi_{0}: \mathcal{C}(\Omega, \mathbb{R}) \rightarrow \mathcal{A}$ be an algebra homomorphism preserving units. Then there exists a unique (complex) algebra homomorphism $\Phi: \mathcal{C}(\Omega) \rightarrow \mathcal{A}$ such that $\left.\Phi\right|_{\mathcal{C}(\Omega, \mathbb{R})}=\Phi_{0}$. Moreover, if $\Phi_{0}$ is continuous, then so is $\Phi$.

Proof. Let $\Phi(f):=\Phi_{0}(\operatorname{Re} f)+i \Phi_{0}(\operatorname{Im} f)(f \in \mathcal{C}(\Omega))$. It is easy to check that $\Phi$ is a unique homomorphic extension of $\Phi_{0}$; moreover, the extension preserves continuity.

1.13. THEOREM (the homomorphism theorem). Let $\Omega$ be a compact Hausdorff space. If $\Phi: \mathcal{C}(\Omega) \rightarrow \mathcal{B}(\mathcal{H})$ is a continuous algebra homomorphism preserving units, then:

(a) there exists a closed subset $A \subset \Omega$ such that $\operatorname{ker} \Phi=\{f \in \mathcal{C}(\Omega)$ : $\left.\left.f\right|_{A} \equiv 0\right\}$,

(b) there exists an algebra monomorphism $\Phi_{0}: \mathcal{C}(A) \rightarrow \mathcal{B}(\mathcal{H})$, which preserves units and is a homeomorphism onto its range, such that $\Phi(f)=$ $\Phi_{0}\left(\left.f\right|_{A}\right)$ for $f \in \mathcal{C}(\Omega)$,

(c) $\sigma(\Phi(f))=f(A)$ for $f \in \mathcal{C}(\Omega)$,

(d) the range of $\Phi$ is closed,

(e) $\Phi$ is an open mapping between its domain and its range,

(f) $A$ and the carrier space of the range of $\Phi$ are homeomorphic,

(g) $\Phi(f)$ is scalar for $f \in \mathcal{C}(\Omega)$,

(h) $\Phi(\bar{f})=(\Phi(f))^{(*)}$ for $f \in \mathcal{C}(\Omega)$,

(i) there exists $G \in \mathcal{G}(\mathcal{H})$ such that $G^{-1} \Phi(f) G$ is normal for $f \in \mathcal{C}(\Omega)$,

(j) there exist a *-representation $\pi: \mathcal{C}(\Omega) \rightarrow \mathcal{B}(\mathcal{H})$ (preserving units) and $G \in \mathcal{G}(\mathcal{H})$ such that $\Phi(f)=G^{-1} \pi(f) G$ for $f \in \mathcal{C}(\Omega)$.

Proof. First we assume that $\Phi$ is a monomorphism. This means that (a) holds for $A:=\Omega$. We put $\Phi_{0}:=\Phi$. By Theorem 1.10, there exists a spectral measure $E: \mathfrak{B}(\Omega) \rightarrow \mathcal{B}(\mathcal{H})$ such that

$$
\Phi(f)=\int_{\Omega} f d E, \quad f \in \mathcal{C}(\Omega) .
$$

Further, by Theorem 1.5, there exists $G \in \mathcal{G}(\mathcal{H})$ such that $G^{-1} E(\sigma) G$ is an orthogonal projection for $\sigma \in \mathfrak{B}(\Omega)$. Let $F$ denote the nonnegative spectral 
measure given by

$$
F(\sigma):=G^{-1} E(\sigma) G, \quad \sigma \in \mathfrak{B}(\Omega) .
$$

Then

$$
G^{-1} \Phi(f) G=\int_{\Omega} f d F, \quad f \in \mathcal{C}(\Omega) .
$$

Hence we get (g), (i) and (j). Moreover

$\Phi(\bar{f})=G \cdot\left(\int_{\Omega} \bar{f} d F\right) \cdot G^{-1}=G \cdot\left(\int_{\Omega} f d F\right)^{*} \cdot G^{-1}=\left(G \cdot\left(\int_{\Omega} f d F\right) \cdot G^{-1}\right)^{(*)}$.

This gives (h). Furthermore, if $\mathcal{F}:=\left\{G^{-1} \Phi(f) G: f \in \mathcal{C}(\Omega)\right\}$, then $\mathcal{F}$ is an abelian *-algebra with unit and the mapping $\Psi: \mathcal{C}(\Omega) \ni f \mapsto G^{-1} \Phi(f) G$ $\in \mathcal{F}$ is a $*$-algebra isomorphism preserving units. Hence $\Psi$ is an isometry, $\mathcal{F}$ is closed and $\Psi$, considered as a homomorphism between $\mathcal{C}(\Omega)$ and $\mathcal{B}(\mathcal{H})$, preserves spectra (since each noninvertible element of $\mathcal{C}(\Omega)$ is a topological divisor of zero). This gives (b) (and (e) as well), (c) and (d). The property (f) follows from (b) and (d).

Now let $\Phi$ be any continuous homomorphism. By Theorem 1.11 there exist a closed subset $A \subset \Omega$ and a continuous algebra monomorphism preserving units $\Phi_{0}: \mathcal{C}(A) \rightarrow \mathcal{B}(\mathcal{H})$ such that $\operatorname{ker} \Phi=\left\{f \in \mathcal{C}(\Omega):\left.f\right|_{A} \equiv 0\right\}$ and $\Phi(f)=\Phi_{0}\left(\left.f\right|_{A}\right)$ for $f \in \mathcal{C}(\Omega)$. It is easy to check that $\Phi$ satisfies all conditions (a)-(j) ((e) follows from the open mapping theorem).

1.14. Corollary. Let $K \subset \mathbb{C}$ be a (nonempty) compact subset and $\varphi: K \ni z \mapsto z \in \mathbb{C}$. Let $\Phi, \Psi: \mathcal{C}(K) \rightarrow \mathcal{B}(\mathcal{H})$ be continuous algebra homomorphisms preserving units. If $\Phi(\varphi)=\Psi(\varphi)$, then $\Phi=\Psi$.

Proof. Theorem 1.13 says that $\Phi(\bar{\varphi})=\Psi(\bar{\varphi})\left(^{3}\right)$. Thus the conclusion follows from the Stone-Weierstrass theorem.

2. Scalar operators. In this section we give a characterization of scalar operators. First we give some of their properties which are simple generalizations of the properties of normal operators.

2.1. Proposition. For a scalar operator $A \in \mathcal{B}(\mathcal{H})$ the following conditions hold true:

(1) if $\sigma(A)=\{0\}$, then $A=0$,

(2) $A^{*}$ is scalar,

(3) $p(A)$ is scalar for every $p \in \mathbb{C}[Z]$,

(4) if $A \in \mathcal{G}(\mathcal{H})$, then $A^{-1}$ is scalar,

(5) for every $G \in \mathcal{G}(\mathcal{H}), G^{-1} A G$ is scalar,

(6) for every $B \in\{A\}_{C C}, B$ is scalar $\left({ }^{4}\right)$.

\footnotetext{
$\left({ }^{3}\right)$ This follows from the uniqueness of the quasi-adjoint of a scalar operator.

$\left({ }^{4}\right) X_{C C}$ denotes the double commutant of a set $X$.
} 
2.2. Proposition. Let $A \in \mathcal{B}(\mathcal{H})$ be a scalar operator. Then:

(1) (Fuglede's type theorem) $A^{(*)} \in\{A\}_{C C}$,

(2) for every $G \in \mathcal{G}(\mathcal{H}),\left(G^{-1} A G\right)^{(*)}=G^{-1} A^{(*)} G$,

(3) for every $\lambda \in \mathbb{C},(\lambda A)^{(*)}=\bar{\lambda} A^{(*)}$,

(4) $A^{(*)}$ is scalar,

(5) $\left(A^{(*)}\right)^{(*)}=A$,

(6) $\left(A^{(*)}\right)^{*}=\left(A^{*}\right)^{(*)}$,

(7) there exists $G \in \mathcal{G}_{+}(\mathcal{H})$ such that $A^{(*)}=G^{-1} A^{*} G$,

(8) (Putnam's type theorem) if moreover $B$ is a scalar operator, $X \in$ $\mathcal{B}(\mathcal{H})$ and $X A=B X$, then $X A^{(*)}=B^{(*)} X$.

Proof. Condition (1) follows from the Fuglede theorem; conditions (2)-(6) are easily seen to be true.

(7) Let $S \in \mathcal{G}(\mathcal{H})$ be such that $N:=S A S^{-1}$ is normal. Then

$$
A^{(*)}=S^{-1} N^{*} S, \quad A^{*}=S^{*} N^{*}\left(S^{*}\right)^{-1},
$$

and hence

$$
A^{(*)}=\left(S^{*} S\right)^{-1} A^{*}\left(S^{*} S\right) .
$$

Thus $G:=S^{*} S$ gives the required similarity.

(8) Let $N$ and $M$ be normal operators and $G_{1}, G_{2} \in \mathcal{G}(\mathcal{H})$ be such that $A=G_{1} M G_{1}^{-1}$ and $B=G_{2} N G_{2}^{-1}$. Then, since $X A=B X$, we have

$$
\left(G_{2}^{-1} X G_{1}\right) M=N\left(G_{2}^{-1} X G_{1}\right) .
$$

Applying the Putnam theorem we obtain

$$
\left(G_{2}^{-1} X G_{1}\right) M^{*}=N^{*}\left(G_{2}^{-1} X G_{1}\right) .
$$

This completes the proof.

2.3. Theorem. Let $M$ be a scalar operator. Then there exist $U, A \in$ $\mathcal{B}(\mathcal{H})$ such that:

(i) $U, A \in\{M\}_{C C}$,

(ii) $U$ is similar to a unitary operator and $\left.U\right|_{\mathcal{N}(M)}=I_{\mathcal{N}(M)}\left({ }^{5}\right)$,

(iii) $A$ is similar to a nonnegative operator, $\mathcal{N}(A)=\mathcal{N}(M)$ and $A^{2}=$ $M^{(*)} M$,

(iv) $M=U A(=A U)$.

Moreover, if $M$ is invertible and $M=U_{1} A_{1}$, where $U_{1}$ is similar to a unitary operator, $A_{1}$ is similar to a selfadjoint one, and $U_{1}$ and $A_{1}$ commute, then $U_{1}=U$ and $A_{1}=A$.

Proof. There exist a normal operator $N$ and $G \in \mathcal{G}(\mathcal{H})$ such that $M=$ $G N G^{-1}$. Let $N=Q B$ be the polar decomposition of $N$. Since $N$ is normal, $Q, B \in\{N\}_{C C}$. Put $V:=\left.Q\right|_{\overline{\mathcal{R}(N)}} \oplus I_{\mathcal{N}(N)}$. Then $V$ is a unitary operator such

$\left(^{5}\right) \mathcal{N}(R)$ denotes the kernel of $R \in \mathcal{B}(\mathcal{H})$ and $\mathcal{R}(R)$ its range. 
that $N=V B$ and $\left.V\right|_{\mathcal{N}(N)}=I_{\mathcal{N}(N)}$. Let $P$ denote the orthogonal projection onto $\mathcal{N}(N)$. Then $P$ also belongs to the double commutant of $N$, which in turn implies that $V \in\{N\}_{C C}$. Set $U:=G V G^{-1}$ and $A:=G B G^{-1}$. Then $U$ and $A$ satisfy all the requirements.

Now suppose that $M$ is invertible and $U_{1}$ and $A_{1}$ are as in the statement of the theorem. Since $A_{1}$ and $U_{1}$ commute, $M$ commutes with $U_{1}$ and $A_{1}$. Moreover, $M$ is invertible and so are $U, U_{1}, A, A_{1}\left({ }^{6}\right)$. But $U, A \in\{M\}_{C C}$, so $U$ commutes with $U_{1}$ and $A$ with $A_{1}$. Moreover, $U^{-1} U_{1}=A A_{1}^{-1}$. It remains to show that $U^{-1} U_{1}=I$. Put $U_{0}:=U^{-1} U_{1}$ and $A_{0}:=A A_{1}^{-1}$. We know that $\sigma(U) \cup \sigma\left(U_{1}\right) \subset \mathbb{T}$ and $\sigma(A) \cup \sigma\left(A_{1}\right) \subset \mathbb{R}_{+}\left({ }^{7}\right)$. By commutativity we get $\sigma\left(U_{0}\right) \subset \mathbb{T}$ and $\sigma\left(A_{0}\right) \subset \mathbb{R}_{+}$(for commuting operators $C$ and $D$ we have $\sigma(C D) \subset \sigma(C) \cdot \sigma(D)$, by the properties of the Gelfand transform). But $U_{0}=A_{0}$, so $\sigma\left(U_{0}\right)=\{1\}$. Since $U^{-1}$ and $U_{1}$ are scalar and commute, we deduce from Theorem 1.6 that $U_{0}$ is also scalar. But $\sigma\left(U_{0}\right)=\{1\}$, so $U_{0}=I$.

It follows from Theorem 1.6 that each operator for which there exist $U, A \in \mathcal{B}(\mathcal{H})$ as in the above theorem, is scalar. In the particular case when this operator is invertible, such a decomposition is unique and we call it the quasi-polar decomposition of the invertible operator.

The following result is a consequence of Theorem 1.3.

2.4. Corollary. If $A \in \mathcal{B}(\mathcal{H})$, then the following conditions are equivalent:

(1) A is similar to a selfadjoint operator,

(2) $\sup _{t \in \mathbb{R}}\|\exp (i t A)\|<\infty$,

(3) there exists $t \in(0, \infty)$ such that $\|A\|<\pi / t$ and

$$
\sup _{k \in \mathbb{Z}}\|\exp (i k t A)\|<\infty,
$$

(4) $\sigma(A) \subset \mathbb{R}$ and

$$
\sup _{\substack{\lambda \in \mathbb{C} \backslash \mathbb{R} \\ k \in \mathbb{Z}}}\left\|\left[(A-\lambda)(A-\bar{\lambda})^{-1}\right]^{k}\right\|<\infty
$$

(5) there exists $\lambda \in \mathbb{C} \backslash \mathbb{R}$ such that $\lambda, \bar{\lambda} \in \varrho(A)\left(^{8}\right)$ and

$$
\sup _{k \in \mathbb{Z}}\left\|\left[(A-\lambda)(A-\bar{\lambda})^{-1}\right]^{k}\right\|<\infty .
$$

Proof. The implications $(1) \Rightarrow(2) \Rightarrow(3),(1) \Rightarrow(4) \Rightarrow(5)$ are obvious (they hold for selfadjoint operators).

\footnotetext{
$\left({ }^{6}\right)$ Here the fact that the operators commute is important.

$\left({ }^{7}\right)$ Here $\mathbb{T}:=\{z \in \mathbb{C}:|z|=1\}$.

$\left(^{8}\right) \varrho(R)$ denotes the resolvent set of $R \in \mathcal{B}(\mathcal{H})$.
} 
$(3) \Rightarrow(1)$. Set $U:=\exp (i t A)$. Then, by Theorem $1.3, U$ is similar to a unitary operator, so $\sigma(U) \subset \mathbb{T}$. Hence $\sigma(A) \subset \mathbb{R}$. But $\|i t A\|<\pi$, so $\sigma(t A) \subset(-\pi, \pi)$. This gives $\sigma(U) \subset \mathbb{T} \backslash\{-1\}$. If $D:=\mathbb{C} \backslash\{t \in \mathbb{R}: t \leq 0\}$, then there exists a holomorphic mapping $\log : D \rightarrow \mathbb{C}$ such that $\log 1=$ 0 and $\exp \circ \log =\mathrm{id}_{D}$. Then, by the Dunford-Riesz functional calculus, $\log U=i t A$. Hence it $A$ is similar to a selfadjoint operator and so is $A$ $(t \neq 0)$.

$(5) \Rightarrow(1)$. Set $U:=(A-\lambda)(A-\bar{\lambda})^{-1}$. Then, by Theorem 1.3, $U$ is similar to a unitary operator. Hence $A$ is similar to a selfadjoint one because $A=$ $(\lambda-\bar{\lambda} U)(1-U)^{-1}$.

Now we can state the characterization of scalar operators.

2.5. Theorem. If $A \in \mathcal{B}(\mathcal{H})$, then the following conditions are equivalent:

(1) A is a scalar operator,

(2) there exists a (nonempty) compact Hausdorff space $\Omega$ and a continuous algebra homomorphism $\Phi: \mathcal{C}(\Omega) \rightarrow \mathcal{B}(\mathcal{H})$ preserving units such that $A \in \Phi(\mathcal{C}(\Omega))$,

(3) there exists a continuous algebra homomorphism $\Phi: \mathcal{C}(\sigma(A)) \rightarrow$ $\mathcal{B}(\mathcal{H})$ preserving units such that $\Phi\left(\mathrm{id}_{\sigma(A)}\right)=A$,

(4) there exists a spectral measure $E: \mathfrak{B}(\sigma(A)) \rightarrow \mathcal{B}(\mathcal{H})$ such that $A=$ $\int_{\sigma(A)} z E(d z)$,

(5) there exists $B \in \mathcal{B}(\mathcal{H})$ such that $A B=B A$ and

$$
\sup _{z \in \mathbb{C}}\|\exp (z A-\bar{z} B)\|<\infty
$$

(6) there exist $B \in \mathcal{B}(\mathcal{H})$ and $p, q \in(0, \infty)$ such that $A B=B A,\|A+B\|$ $<\pi / p,\|A-B\|<\pi / q$ and

$$
\sup _{k \in \mathbb{Z}}\|\exp [i k p(A+B)]\|<\infty, \quad \sup _{k \in \mathbb{Z}}\|\exp [k q(A-B)]\|<\infty,
$$

(7) there exists $B \in \mathcal{B}(\mathcal{H})$ such that

$$
\sup _{z \in \mathbb{C}}\|\exp (z A) \cdot \exp (-\bar{z} B)\|<\infty
$$

(8) there exist $B \in \mathcal{B}(\mathcal{H})$ and $\lambda \in \mathbb{C} \backslash \mathbb{R}$ such that $A B=B A, \lambda, \bar{\lambda} \in$ $\varrho(A+B) \cap \varrho(i(A-B))$ and

$$
\begin{array}{r}
\sup _{k \in \mathbb{Z}}\left\|\left[(A+B-\lambda)(A+B-\bar{\lambda})^{-1}\right]^{k}\right\|<\infty, \\
\sup _{k \in \mathbb{Z}}\left\|\left[(i(A-B)-\lambda)(i(A-B)-\bar{\lambda})^{-1}\right]^{k}\right\|<\infty .
\end{array}
$$

Moreover, if $A$ is scalar, then the operator $B$ which appears in each of (5)-(8) is unique and $B=A^{(*)}\left({ }^{9}\right)$.

$\left({ }^{9}\right)$ In particular, $B$ in condition (7) commutes with $A$. 
Proof. The equivalences $(1) \Leftrightarrow(2) \Leftrightarrow(3) \Leftrightarrow(4)$ can be deduced from the theorems of Section 1 and properties of normal operators.

The implications $(1) \Rightarrow(5) \Rightarrow(6),(1) \Rightarrow(7),(1) \Rightarrow(8)$ are clear.

$(6) \Rightarrow(1)$. As in the proof of Corollary 2.4 we see that the operators $A+B$ and $(A-B) / i$ are similar to selfadjoint ones. This means that $(A+B)^{(*)}=$ $A+B$ and $((A-B) / i)^{(*)}=(A-B) / i$. Since $A$ and $B$ commute, $A$ is scalar and

$$
A^{(*)}=\left(\frac{A+B}{2}\right)^{(*)}-i\left(\frac{A-B}{2 i}\right)^{(*)}=\frac{A+B}{2}-i \frac{A-B}{2 i}=B .
$$

This gives (1) and the uniqueness of $B$.

$(7) \Rightarrow(1)$. It suffices to prove that $B$ and $A$ commute (then we apply (6)). Consider the mapping

$$
\varphi: \mathbb{C} \ni z \mapsto \exp (z A) \cdot B \cdot \exp (-z A) \in \mathcal{B}(\mathcal{H}) .
$$

It is an entire function which is bounded because

$$
\begin{aligned}
\|\varphi(z)\| & =\|\exp (z A) \cdot \exp (-\bar{z} B) \cdot B \cdot \exp (\bar{z} B) \cdot \exp (-z A)\| \\
& \leq\|\exp (z A) \cdot \exp (-\bar{z} B)\| \cdot\|B\| \cdot\|\exp (\bar{z} B) \cdot \exp (-z A)\| \\
& \leq\left(\sup _{z \in \mathbb{C}}\|\exp (z A) \cdot \exp (-\bar{z} B)\|\right)^{2} \cdot\|B\|<\infty, \quad z \in \mathbb{C} .
\end{aligned}
$$

Hence, by the Liouville theorem, $\varphi$ is constant. This means that

$$
\exp (z A) \cdot B=B \cdot \exp (z A), \quad z \in \mathbb{C} .
$$

So the coefficients of both power series coincide and hence $A B=B A$.

The proof of $(8) \Rightarrow(1)$ is similar to that of $(6) \Rightarrow(1)$.

If $K \subset \mathbb{C}$ is an infinite compact subset, then we denote by $\mathcal{R}_{0}(K)$ the algebra of rational functions with poles off $K$ and consider it as a subalgebra of $\mathcal{C}(K)$ with the supremum norm $\|\cdot\|_{K} \cdot \mathcal{R}(K)$ denotes the uniform closure of $\mathcal{R}_{0}(K)$.

2.6. Corollary. Let $A \in \mathcal{B}(\mathcal{H})$ with $\operatorname{card} \sigma(A) \geq \aleph_{0}$ and $K:=\sigma(A)$. If $\mathcal{R}(K)=\mathcal{C}(K)$, then the following conditions are equivalent:

(1) A is scalar,

(2) there exists a constant $M>0$ such that

$$
\|W(A)\| \leq M\|W\|_{K}, \quad W \in \mathcal{R}_{0}(K) .
$$

Proof. The implication $(1) \Rightarrow(2)$ is clear.

$(2) \Rightarrow(1)$. The condition (2) tells us that the mapping

$$
\mathcal{R}_{0}(K) \ni W \mapsto W(A) \in \mathcal{B}(\mathcal{H})
$$

is a continuous algebra homomorphism preserving units. So it can be extended to a continuous algebra homomorphism between $\mathcal{C}(K)$ and $\mathcal{B}(\mathcal{H})\left({ }^{10}\right)$. Now it remains to apply Theorem 2.5.

\footnotetext{
$\left({ }^{10}\right)$ Since $\mathcal{R}(K)=\mathcal{C}(K)$.
} 
2.7. TheOREM. The following conditions hold true:

(1) each projection is a scalar operator,

(2) for $A \in \mathcal{B}(\mathcal{H})$ with $\sigma(A)=\left\{\lambda_{1}, \ldots, \lambda_{n}\right\}$, where $\lambda_{1}, \ldots, \lambda_{n}$ are distinct, the following conditions are equivalent:

(a) $A$ is a scalar operator,

(b) $\left(A-\lambda_{1}\right) \ldots\left(A-\lambda_{n}\right)=0$,

(c) there exist $P_{1}, \ldots, P_{n} \in \mathcal{B}(\mathcal{H})$ such that $P_{j} P_{k}=\delta_{j k} P_{j}$ for all $j, k \in\{1, \ldots, n\}, P_{1}+\ldots+P_{n}=I$ and $A=\lambda_{1} P_{1}+\ldots+\lambda_{n} P_{n}$.

Proof. (1) follows easily from Theorem 1.4.

$(2),(b) \Leftrightarrow(c)$. This is a well known theorem of the theory of Banach algebras (see [B-D, Proposition I.8.10] for the proof of (b) $\Rightarrow(\mathrm{c})$, the converse implication is a simple algebraic calculation).

The implication $(\mathrm{a}) \Rightarrow(\mathrm{c})$ is true for normal operators, hence also for scalar ones; (c) $\Rightarrow$ (a) follows from (1) and Theorem 1.6.

For more results concerning similarity to a unitary or selfadjoint operator see also [Nab, Ca1, Ca2, G-K, B-N].

2.8. Theorem. Any scalar operator $A$ is the norm limit of a sequence of scalar operators belonging to the double commutant of $A$ each of which has a finite spectrum.

Proof. Normal operators have this property.

2.9. Proposition. If $A \in \mathcal{B}\left(\mathbb{C}^{n}\right)(n \geq 1)$ and card $\sigma(A)=n$, then $A$ is a scalar operator.

Proof. By Jordan theory, $A$ is similar to a diagonal normal operator.

\section{Abelian families of operators}

3.1. Definition. Families $\mathcal{S}, \mathcal{T} \subset \mathcal{B}(\mathcal{H})$ are said to be similar if there exists $G \in \mathcal{G}(\mathcal{H})$ such that

$$
\begin{array}{ll}
G^{-1} S G \in \mathcal{T}, & S \in \mathcal{S}, \\
G T G^{-1} \in \mathcal{S}, & T \in \mathcal{T} .
\end{array}
$$

If $A, B \in \mathcal{B}(\mathcal{H})$, then $A$ and $B$ are similar if $\{A\}$ and $\{B\}$ are similar.

3.2. Definition. Let $\mathcal{S} \subset \mathcal{B}(\mathcal{H})$. We say that the set $\mathcal{S}$ is:

- abelian if all elements of $\mathcal{S}$ commute,

- separately scalar if each element of $\mathcal{S}$ is a scalar operator,

- jointly scalar if $\mathcal{S}$ is similar to a set of normal operators.

In other words:

$\mathcal{S}$ is separately scalar $\Leftrightarrow \forall S \in \mathcal{S} \exists G \in \mathcal{G}(\mathcal{H}): G^{-1} S G$ is normal,

$\mathcal{S}$ is jointly scalar $\Leftrightarrow \exists G \in \mathcal{G}(\mathcal{H}) \forall S \in \mathcal{S}: G^{-1} S G$ is normal. 
3.3. Definition. Let $\mathcal{A} \subset \mathcal{B}(\mathcal{H})$. We say that:

- $\mathcal{A}$ is a "quasi-star" algebra (for short: $(*)$-algebra) if it is a subalgebra of $\mathcal{B}(\mathcal{H})$ which is separately scalar and invariant under the operation of taking quasi-adjoints,

- $\mathcal{A}$ is a $\mathcal{C}^{(*)}$-algebra if it is a closed (*)-algebra.

A subalgebra of $\mathcal{B}(\mathcal{H})$ is called an algebra with unit if it contains the unit of $\mathcal{B}(\mathcal{H})$.

3.4. THEOREM. Let $\mathcal{S} \subset \mathcal{B}(\mathcal{H})$ be any abelian jointly scalar set and $G \in \mathcal{G}(\mathcal{H})$ be such that $G^{-1} S G$ is normal for every $S \in \mathcal{S}$. Then $G^{-1} S G$ is normal for every $S \in \mathcal{S}_{C C}$ and $\mathcal{S}_{C C}$ is a commutative $\mathcal{C}^{(*)}$-algebra with unit containing $\mathcal{S}$.

Proof. Set $\mathcal{N}:=\left\{G^{-1} S G: S \in \mathcal{S}\right\}$. By assumptions, $\mathcal{N}$ is an abelian set of normal operators. Hence $\mathcal{N}_{C C}$ is a commutative $\mathcal{C}^{*}$-algebra (in particular, its elements are normal operators) with unit, containing $\mathcal{N}$. But $\mathcal{N}_{C C}=$ $G^{-1} \cdot \mathcal{S}_{C C} \cdot G$, which completes the proof.

3.5. Corollary. (a) Any abelian separately scalar set is contained in an abelian $(*)$-algebra with unit.

(b) If $\mathcal{A}$ is an abelian (*)-algebra, then $(*)$ is an involution on $\mathcal{A}$.

Proof. (a) Let $\mathcal{S}$ be any abelian separately scalar set. We conclude from Theorems 1.6 and 3.4 that for all $A_{1}, \ldots, A_{n} \in \mathcal{S}\left(n \in \mathbb{N}_{*}\right)$ the double commutant of the set $\left\{A_{1}, \ldots, A_{n}\right\}$ is a commutative (*)-algebra with unit which is contained in the commutant of $\mathcal{S}$. Hence the set

$$
\bigcup_{\substack{A_{1}, \ldots, A_{n} \in \mathcal{S} \\ n \geq 1}}\left\{A_{1}, \ldots, A_{n}\right\}_{C C}
$$

is an abelian $(*)$-algebra with unit containing $\mathcal{S}$.

(b) By (a) it remains to show that if $A$ and $B$ are commuting scalar operators, then

$$
(A+B)^{(*)}=A^{(*)}+B^{(*)} \text { and }(A B)^{(*)}=A^{(*)} B^{(*)} .
$$

By Proposition 2.2(1), (4), the set $\left\{A, B, A^{(*)}, B^{(*)}\right\}$ is abelian and separately scalar. Hence, by Theorem 1.6, there exists $G \in \mathcal{G}(\mathcal{H})$ such that the operators $G^{-1} A G, G^{-1} B G, G^{-1} A^{(*)} G$ and $G^{-1} B^{(*)} G$ are normal and commuting. Therefore the operators $G^{-1}(A+B) G, G^{-1} A B G, G^{-1}\left(A^{(*)}+B\right) G$ and $G^{-1} A^{(*)} B G$ are also normal and consequently (by the definition of the quasi-adjoint)

$$
\begin{aligned}
(A+B)^{(*)} & =G\left(G^{-1}(A+B) G\right)^{*} G^{-1} \\
& =G\left(G^{-1} A G\right)^{*} G^{-1}+G\left(G^{-1} B G\right)^{*} G^{-1}=A^{(*)}+B^{(*)},
\end{aligned}
$$




$$
\begin{aligned}
(A B)^{(*)} & =\left(G^{-1} A B G\right)^{*} G^{-1}=G\left[\left(G^{-1} A G\right)\left(G^{-1} B G\right)\right]^{*} G^{-1} \\
& =G\left(G^{-1} B G\right)^{*}\left(G^{-1} A G\right)^{*} G^{-1} \\
& =\left[G\left(G^{-1} B G\right)^{*} G^{-1}\right] \cdot\left[G\left(G^{-1} A G\right)^{*} G^{-1}\right]=B^{(*)} A^{(*)}=A^{(*)} B^{(*)},
\end{aligned}
$$

which completes the proof.

It follows from Corollary 3.5 that for any abelian separately scalar set $\mathcal{S} \subset \mathcal{B}(\mathcal{H})$ there exists the smallest (not necessarily closed) subalgebra with unit which is an abelian $(*)$-algebra containing $\mathcal{S}$. We denote it by $(*)-\operatorname{Alg}(\mathcal{S})$ and call it the $(*)$-algebra generated by $\mathcal{S}$. For a scalar operator $A$ we write $(*)-\operatorname{Alg}(A)$ instead of $(*)-\operatorname{Alg}(\{A\})$.

We shall see below that in general the (norm) closure of an abelian $(*)$ algebra may not be separately scalar (see Remark 4.11). This is the reason why we do not define the $\mathcal{C}^{(*)}$-algebra generated by an abelian separately scalar set.

The following fact belongs to folklore knowledge. For the reader's convenience we sketch its proof.

3.6. Theorem. Let $(\mathcal{H},\langle\cdot,-\rangle)$ be a Hilbert space and $\mathcal{S} \subset \mathcal{B}(\mathcal{H})$. Then the following conditions are equivalent:

(1) $\mathcal{S}$ is jointly scalar,

(2) there exists $A \in \mathcal{G}_{+}(\mathcal{H})$ such that $A^{-1} S A$ is normal for any $S \in \mathcal{S}$,

(3) there exists an equivalent scalar product $\langle\cdot,-\rangle_{\mathcal{S}}$ on $\mathcal{H}$ such that each element of $\mathcal{S}$ is a normal operator in the Hilbert space $\left(\mathcal{H},\langle\cdot,-\rangle_{\mathcal{S}}\right)$.

Proof. The implication $(2) \Rightarrow(1)$ is clear.

$(1) \Rightarrow(2)$. Let $G \in \mathcal{G}(\mathcal{H})$ be such that $G^{-1} S G$ is normal for any $S \in \mathcal{S}$. Let $G^{-1}=U B$ be the polar decomposition; then $U$ is a unitary operator and $B \in \mathcal{G}_{+}(\mathcal{H})$ (because $G$ is invertible).

We shall show that $A:=B^{-1}$ is a nonnegative operator realizing the similarity. Since $A \in \mathcal{G}_{+}(\mathcal{H})$, it remains to show that $A^{-1} S A$ is normal for any $S \in \mathcal{S}$. Indeed, $G^{-1} S G$ is normal and $A^{-1} S A=U^{-1}\left(G^{-1} S G\right) U$, which gives (2) because $U$ is unitary.

$(2) \Rightarrow(3)$. Let $A \in \mathcal{G}_{+}(\mathcal{H})$ be as in (2). Define

$$
\langle x, y\rangle_{\mathcal{S}}:=\left\langle A^{-2} x, y\right\rangle, \quad x, y \in \mathcal{H} .
$$

Since $A \in \mathcal{G}_{+}(\mathcal{H}),\langle\cdot,-\rangle_{\mathcal{S}}$ is equivalent to $\langle\cdot,-\rangle$ and it remains to prove that each $S \in \mathcal{S}$ is normal in $\left(\mathcal{H},\langle\cdot,-\rangle_{\mathcal{S}}\right)$. Clearly $S$ is $\|\cdot\|_{\mathcal{S}}$-bounded. We know that $A^{-1} S A$ is normal, so the operators $A^{-1} S A$ and $A S^{*} A^{-1}=\left(A^{-1} S A\right)^{*}$ commute and consequently so do $S$ and $A^{2} S^{*} A^{-2}$. Therefore it is enough to show that the adjoint of $S$ in $\left(\mathcal{H},\langle\cdot,-\rangle_{\mathcal{S}}\right)$ is $A^{2} S^{*} A^{-2}$. Indeed, for all $x, y \in \mathcal{H}$ we have 


$$
\begin{aligned}
\langle S x, y\rangle_{\mathcal{S}} & =\left\langle A^{-2} S x, y\right\rangle=\left\langle S x, A^{-2} y\right\rangle=\left\langle x, S^{*} A^{-2} y\right\rangle \\
& =\left\langle A^{2} A^{-2} x, S^{*} A^{-2} y\right\rangle=\left\langle A^{-2} x, A^{2} S^{*} A^{-2} y\right\rangle=\left\langle x,\left(A^{2} S^{*} A^{-2}\right) y\right\rangle_{\mathcal{S}}
\end{aligned}
$$

which yields (3).

$(3) \Rightarrow(2)$. Let $\langle\cdot,-\rangle_{\mathcal{S}}$ be as in (3). By the Riesz representation theorem there exists $B \in \mathcal{B}_{+}(\mathcal{H})$ such that

$$
\langle x, y\rangle_{\mathcal{S}}=\langle B x, y\rangle, \quad x, y \in \mathcal{H} .
$$

The equivalence of the scalar products implies that $B \in \mathcal{G}_{+}(\mathcal{H})$ and in consequence $B^{-1} \in \mathcal{G}_{+}(\mathcal{H})$. By the square root theorem there exists $A \in$ $\mathcal{B}_{+}(\mathcal{H})$ such that $A^{2}=B^{-1}$. Since $B$ is invertible, so is $A$. This means that $A \in \mathcal{G}_{+}(\mathcal{H})$. We show that for any $S \in \mathcal{S}$ the operator $A^{-1} S A$ is normal. Indeed, for $x, y \in \mathcal{H}$ we have (by (3-1))

$$
\begin{aligned}
\langle S x, y\rangle_{\mathcal{S}} & =\langle B S x, y\rangle=\left\langle A^{-2} S x, y\right\rangle=\left\langle S x, A^{-2} y\right\rangle=\left\langle x, S^{*} A^{-2} y\right\rangle \\
& =\left\langle A^{2} A^{-2} x, S^{*} A^{-2} y\right\rangle=\left\langle A^{-2} x, A^{2} S^{*} A^{-2} y\right\rangle \\
& =\left\langle B x, A^{2} S^{*} A^{-2} y\right\rangle=\left\langle x, A^{2} S^{*} A^{-2} y\right\rangle_{\mathcal{S}} .
\end{aligned}
$$

Thus $A^{-2} S^{*} A^{2}$ is the adjoint of $S$ in the space $\left(\mathcal{H},\langle\cdot,-\rangle_{\mathcal{S}}\right)$. But $S$ is normal in this space, so $S$ and $A^{2} S^{*} A^{-2}$ commute. This means that $S A^{2} S^{*} A^{-2}=$ $A^{2} S^{*} A^{-2} S$. Finally, we have (due to $A=A^{*}$ )

$$
\begin{aligned}
\left(A^{-1} S A\right)\left(A^{-1} S A\right)^{*} & =A^{-1} S A \cdot A S^{*} A^{-1}=A^{-1}\left(S A^{2} S^{*} A^{-2}\right) A \\
& =A^{-1}\left(A^{2} S^{*} A^{-2} S\right) A=A S^{*} A^{-1} \cdot A^{-1} S A \\
& =\left(A^{-1} S A\right)^{*}\left(A^{-1} S A\right) .
\end{aligned}
$$

Hence $A^{-1} S A$ is normal. This completes the proof.

We now offer a few conditions equivalent to the joint scalarity of abelian separately scalar sets. Observe that if $A$ is scalar, then $A+A^{(*)}$ is similar to a selfadjoint operator (because $\left(A+A^{(*)}\right)^{(*)}=A+A^{(*)}$ ), so its spectrum is real; in particular, the operators $A+A^{(*)}-i$ and $A+A^{(*)}+i$ are invertible. Likewise, $i\left(A-A^{(*)}\right)-i$ and $i\left(A-A^{(*)}\right)+i$ are also invertible.

3.7. TheOREM. Let $\mathcal{S} \subset \mathcal{B}(\mathcal{H})$ be an abelian separately scalar set. The following conditions are equivalent:

(1) $\mathcal{S}$ is jointly scalar,

(2) there exists $M \in(0, \infty)$ such that for all $\lambda_{1}, \ldots, \lambda_{n} \in \mathbb{C}(n \geq 1)$ and for all $A_{1}, \ldots, A_{n} \in \mathcal{S}$,

$$
\left\|\exp \left[\left(\lambda_{1} A_{1}-\bar{\lambda}_{1} A_{1}^{(*)}\right)+\ldots+\left(\lambda_{n} A_{n}-\bar{\lambda}_{n} A_{n}^{(*)}\right)\right]\right\| \leq M,
$$

(3) there exists $M \in(0, \infty)$ such that for all $k_{1}, \ldots, k_{n}, l_{1}, \ldots, l_{n} \in \mathbb{Z}$ $(n \geq 1)$ and for all $A_{1}, \ldots, A_{n} \in \mathcal{S}$, 


$$
\begin{aligned}
\|\left[\left(A_{1}+A_{1}^{(*)}\right)-i\right]^{k_{1}} \cdot\left[\left(A_{1}+A_{1}^{(*)}\right)+i\right]^{-k_{1}} \cdot\left[i\left(A_{1}-A_{1}^{(*)}\right)-i\right]^{l_{1}} \\
\cdot\left[i\left(A_{1}-A_{1}^{(*)}\right)+i\right]^{-l_{1}} \ldots\left[\left(A_{n}+A_{n}^{(*)}\right)-i\right]^{k_{n}} \cdot\left[\left(A_{n}+A_{n}^{(*)}\right)+i\right]^{-k_{n}} \\
\cdot\left[i\left(A_{n}-A_{n}^{(*)}\right)-i\right]^{l_{n}} \cdot\left[i\left(A_{n}-A_{n}^{(*)}\right)+i\right]^{-l_{n}} \| \leq M .
\end{aligned}
$$

Proof. For simplicity, we denote by $c\left(A_{1}, \ldots, A_{n} ; k_{1}, \ldots, k_{n} ; l_{1}, \ldots, l_{n}\right)$ (where $A_{1}, \ldots, A_{n} \in \mathcal{S}$ and $k_{1}, \ldots, k_{n}, l_{1}, \ldots, l_{n} \in \mathbb{Z}(n \geq 1)$ ) the operator whose norm appears in (3). By the comment preceding the statement of the theorem, this operator is well defined.

$(1) \Rightarrow(2)$. Since $\mathcal{S}$ is abelian and jointly scalar, we conclude from Theorem 3.4 that $(*)-\operatorname{Alg}(\mathcal{S})$ is an abelian jointly scalar algebra containing $\mathcal{S}$. Therefore we may assume that $\mathcal{S}$ is a commutative $(*)$-algebra. Then (2) is equivalent to

$$
\sup _{A \in \mathcal{S}}\left\|\exp \left(A-A^{(*)}\right)\right\|<\infty .
$$

Let $A \in \mathcal{A}$. Since $\mathcal{S}$ is a jointly scalar algebra, there exists $G \in \mathcal{G}(\mathcal{H})$ such that $N:=G^{-1} A G$ is normal. Then $A=G N G^{-1}$ and $A^{(*)}=G N^{*} G^{-1}$. The operator $\left(N-N^{*}\right) / i$ is selfadjoint and consequently $\exp \left(N-N^{*}\right)$ is unitary. Hence

$$
\begin{aligned}
\left\|\exp \left(A-A^{(*)}\right)\right\| & =\left\|G \exp \left(N-N^{*}\right) G^{-1}\right\| \\
& \leq\|G\| \cdot\left\|\exp \left(N-N^{*}\right)\right\| \cdot\left\|G^{-1}\right\|=\|G\| \cdot\left\|G^{-1}\right\| .
\end{aligned}
$$

$(2) \Rightarrow(1)$. Set

$$
\begin{aligned}
& \mathcal{F}:=\left\{\exp \left[\left(\lambda_{1} A_{1}-\bar{\lambda}_{1} A_{1}^{(*)}\right)+\ldots+\left(\lambda_{n} A_{n}-\bar{\lambda}_{n} A_{n}^{(*)}\right)\right]:\right. \lambda_{1}, \ldots, \lambda_{n} \in \mathbb{C}, \\
&\left.A_{1}, \ldots, A_{n} \in \mathcal{S}, n \geq 1\right\} .
\end{aligned}
$$

By the commutativity of $\mathcal{S}$ and Proposition 2.2(1), $\mathcal{F}$ is an abelian subgroup of $\mathcal{G}(\mathcal{H})$. Moreover, by $(2), \mathcal{F}$ is bounded. It follows from Theorem 1.3 that $\mathcal{F}$ is similar to an abelian group of unitary operators. Let $G \in \mathcal{G}(\mathcal{H})$ be such that $G^{-1} T G$ is unitary for every $T \in \mathcal{F}$. Take $A \in \mathcal{S}$. It suffices to show that $G^{-1} A G$ is normal.

By the definition of $\mathcal{F}$ we have $\exp \left(z A-\bar{z} A^{(*)}\right) \in \mathcal{F}$ for $z \in \mathbb{C}$. Hence $G^{-1} \exp \left(z A-\bar{z} A^{(*)}\right) G$ is unitary for $z \in \mathbb{C}$. The operator $i\left(A+A^{(*)}\right)$ (resp. $\left.A-A^{(*)}\right)$ is the strong generator $\left({ }^{11}\right)$ of the group $\left\{\exp \left(i t A-\overline{i t} A^{(*)}\right)\right\}_{t \in \mathbb{R}}$ (resp. $\left.\left\{\exp \left(t A-\bar{t} A^{(*)}\right)\right\}_{t \in \mathbb{R}}\right)$. So $i G^{-1}\left(A+A^{(*)}\right) G\left(\right.$ resp. $\left.G^{-1}\left(A-A^{(*)}\right) G\right)$ is the strong generator of the group $\left\{G^{-1} \exp \left(i t A-\overline{i t} A^{(*)}\right) G\right\}_{t \in \mathbb{R}}$ (resp. $\left.\left\{G^{-1} \exp \left(t A-\bar{t} A^{(*)}\right) G\right\}_{t \in \mathbb{R}}\right)$ of unitary operators. Thus both $i G^{-1}\left(A+A^{(*)}\right) G$ and $G^{-1}\left(A-A^{(*)}\right) G$ are normal. Moreover, they commute (because $A$ and $A^{(*)}$ commute). Hence $G^{-1} A G=\frac{1}{2}\left[-i \cdot i G^{-1}\left(A+A^{(*)}\right) G+G^{-1}\left(A-A^{(*)}\right) G\right]$ is normal, which gives (1).

\footnotetext{
$\left({ }^{11}\right)$ The relevant convergence is in the norm topology.
} 


$$
\begin{aligned}
&(1) \Rightarrow(3) . \text { Set } \\
& \mathcal{F}:=\left\{c\left(A_{1}, \ldots, A_{n} ; k_{1}, \ldots, k_{n} ; l_{1}, \ldots, l_{n}\right): n \geq 1, A_{1}, \ldots, A_{n} \in \mathcal{S},\right. \\
&\left.k_{1}, \ldots, k_{n}, l_{1}, \ldots, l_{n} \in \mathbb{Z}\right\} .
\end{aligned}
$$

We have to prove that $\mathcal{F}$ is bounded. Let $G \in \mathcal{G}(\mathcal{H})$ be such that $G^{-1} A G$ is normal for all $A \in \mathcal{S}$. Then the operators $G^{-1}\left[\left(A+A^{(*)}-i\right)\left(A+A^{(*)}+i\right)^{-1}\right] G$ and $G^{-1}\left[\left(i\left(A-A^{(*)}\right)-i\right)\left(i\left(A-A^{(*)}\right)+i\right)^{-1}\right] G$ are unitary $(A \in \mathcal{S})$. Now it is easy to check that the set $G^{-1} \cdot \mathcal{F} \cdot G$ consists of unitary operators. This means that $\mathcal{F}$ is bounded.

$(3) \Rightarrow(1)$. Let $\mathcal{F}$ be as in the proof of $(1) \Rightarrow(3)$. It is clear that $\mathcal{F}$ is an abelian subgroup of $\mathcal{G}(\mathcal{H})$. Moreover, by $(3), \mathcal{F}$ is bounded. Applying Theorem 1.3, we get an operator $G \in \mathcal{G}(\mathcal{H})$ such that $G^{-1} T G$ is unitary for $T \in \mathcal{F}$. In particular, $G^{-1}\left[\left(A+A^{(*)}-i\right)\left(A+A^{(*)}+i\right)^{-1}\right] G$ and $G^{-1}\left[\left(i\left(A-A^{(*)}\right)-i\right)\left(i\left(A-A^{(*)}\right)+i\right)^{-1}\right] G$ are unitary operators $(A \in \mathcal{S})$. This means that the operators $G^{-1}\left(A+A^{(*)}\right) G$ and $i G^{-1}\left(A-A^{(*)}\right) G$ are selfadjoint and commute for all $A \in \mathcal{S}$ (because we know the inverse of the Cayley transform). Hence

$$
G^{-1} A G=\frac{G^{-1}\left(A+A^{(*)}\right) G}{2}-i \frac{i G^{-1}\left(A-A^{(*)}\right) G}{2}
$$

is normal, which completes the proof.

Theorem 3.7 is based on the following idea: studying joint scalarity can be replaced by studying similarity to a set of selfadjoint operators (by using the operators $A+A^{(*)}$ and $i\left(A-A^{(*)}\right)$ instead of $\left.A\right)$; this problem can be replaced by studying similarity to a set of unitary operators (by using holomorphic mappings which send the real line into the unit circle $\mathbb{T}$ ) and then by applying Theorem 1.3. In part (2) of Theorem 3.7 we used the mapping $\mathbb{C} \ni z \mapsto \exp (i z) \in \mathbb{C}$, while in (3) the Cayley transform, i.e. the mapping $\mathbb{C} \backslash\{-i\} \ni z \mapsto(z-i) /(z+i) \in \mathbb{C}$. Using other holomorphic mappings with the above mentioned property one can obtain other results concerning joint scalarity.

3.8. Remark. An abelian set $\mathcal{S} \subset \mathcal{B}(\mathcal{H})$ is jointly scalar if and only if there exists a mapping $A \mapsto \widehat{A}$ from $\mathcal{S}$ into $\mathcal{B}(\mathcal{H})$ such that

$$
\sup _{\substack{A_{1}, \ldots, A_{n} \in \mathcal{S} \\ \lambda_{1}, \ldots, \lambda_{n} \in \mathbb{C} \\ n \geq 1}}\left\|\exp \left(\lambda_{1} A_{1}\right) \cdot \exp \left(-\bar{\lambda}_{1} \widehat{A}_{1}\right) \cdot \ldots \cdot \exp \left(\lambda_{n} A_{n}\right) \cdot \exp \left(-\bar{\lambda}_{n} \widehat{A}_{n}\right)\right\|<\infty .
$$

Moreover, if $\mathcal{S}$ is jointly scalar, then the mapping $A \mapsto \widehat{A}$ is unique and $\widehat{A}=A^{(*)}(A \in \mathcal{S})$.

Proof. By Theorem 2.5(7) we see that $\mathcal{S}$ is separately scalar and $\widehat{A}=$ $A^{(*)}(A \in \mathcal{S})$. So it remains to apply Theorem 3.7. 
3.9. Corollary. An abelian separately scalar set is jointly scalar if and only if each of its countable (infinite) subsets is jointly scalar.

Proof. This follows from Theorems 3.7 and 1.6.

Now we can state a generalization of Theorem 1.6.

3.10. TheOREM. Let $\mathcal{S}_{1}, \ldots, \mathcal{S}_{m}(m \geq 2)$ be jointly scalar sets and $\mathcal{S}:=$ $\mathcal{S}_{1} \cup \ldots \cup \mathcal{S}_{m}$. If $\mathcal{S}$ is abelian, then it is jointly scalar.

Proof. Notice that $\mathcal{S}$ is separately scalar and, by assumption, abelian. For any $j \in\{1, \ldots, m\}$ there exists $M_{j} \in(1, \infty)$ such that

$$
\begin{aligned}
\forall n \geq 1, \forall \lambda_{1}, \ldots, \lambda_{n} \in \mathbb{C}, \forall A_{1}, \ldots, A_{n} \in \mathcal{S}_{j}: \\
\left\|\exp \left[\left(\lambda_{1} A_{1}-\bar{\lambda}_{1} A_{1}^{(*)}\right)+\ldots+\left(\lambda_{n} A_{n}-\bar{\lambda}_{n} A_{n}^{(*)}\right)\right]\right\| \leq M_{j}\left({ }^{12}\right) .
\end{aligned}
$$

Take $\lambda_{1}, \ldots, \lambda_{n} \in \mathbb{C}$ and $A_{1}, \ldots, A_{n} \in \mathcal{S}(n \geq 1)$. We may assume that (for some $0 \leq k_{1} \leq \ldots \leq k_{m-1} \leq n$ )

$$
\begin{aligned}
& A_{p} \in \mathcal{S}_{1}, & 1 \leq p \leq k_{1}, \\
\forall j \in\{2, \ldots, m-1\}: & A_{p} \in \mathcal{S}_{j}, & k_{j-1}+1 \leq p \leq k_{j}, \\
& A_{p} \in \mathcal{S}_{m}, & k_{m-1}+1 \leq p \leq n .
\end{aligned}
$$

Then

$$
\begin{aligned}
& \left\|\exp \left[\left(\lambda_{1} A_{1}-\bar{\lambda}_{1} A_{1}^{(*)}\right)+\ldots+\left(\lambda_{n} A_{n}-\bar{\lambda}_{n} A_{n}^{(*)}\right)\right]\right\| \\
& \leq\left\|\exp \left[\left(\lambda_{1} A_{1}-\bar{\lambda}_{1} A_{1}^{(*)}\right)+\ldots+\left(\lambda_{k_{1}} A_{k_{1}}-\bar{\lambda}_{k_{1}} A_{k_{1}}^{(*)}\right)\right]\right\| \ldots \\
& \quad \ldots\left\|\exp \left[\left(\lambda_{k_{m-1}+1} A_{k_{m-1}+1}-\bar{\lambda}_{k_{m-1}+1} A_{k_{m-1}+1}^{(*)}\right)+\ldots+\left(\lambda_{n} A_{n}-\bar{\lambda}_{n} A_{n}^{(*)}\right)\right]\right\| \\
& \quad \leq M_{1} \ldots M_{m}<\infty .
\end{aligned}
$$

Now it suffices to apply Theorem 3.7.

4. Abelian $\mathcal{C}^{(*)}$-algebras. For the proof of the following theorem see [Kat].

4.1. TheOrem (Katznelson). Let $\mathcal{A}$ be a (complex) commutative $B a$ nach algebra with unit. Let $\Omega$ denote the carrier space of the algebra $\mathcal{A}$ and $\Phi: \mathcal{A} \rightarrow \mathcal{C}(\Omega)$ be its Gelfand transform. The following conditions are equivalent:

(1) $\Phi$ is an isomorphism between $\mathcal{A}$ and $\mathcal{C}(\Omega)$,

(2) $\mathcal{A}$ is a semisimple algebra which admits a Hermitian involution and each element of $\mathcal{A}$ with nonnegative spectrum has a square root $($ in $\mathcal{A})$ with nonnegative spectrum.

We state a real version of the Katznelson theorem. As it may be unknown, we give the proof.

\footnotetext{
$\left({ }^{12}\right)$ Because $\mathcal{S}_{j}(j=1, \ldots, n)$ is jointly scalar.
} 
Let $\mathcal{A}$ be a real Banach algebra with unit. To simplify the statements we introduce the following notions:

- $a \in \mathcal{A}$ is invertible if there exists $b \in \mathcal{A}$ such that $a b=b a=1$,

- $a \in \mathcal{A}$ is nonnegative if $a-t$ is invertible for every $t \in(-\infty, 0)$.

4.2. TheOREM. Let $\mathcal{A}$ be a real commutative Banach algebra with unit satisfying the following conditions:

(1) $1+x^{2}$ is invertible in $\mathcal{A}$ for every $x \in \mathcal{A}$,

(2) for every nonnegative $a \in \mathcal{A}$ there exists a nonnegative $b \in \mathcal{A}$ such that $b^{2}=a$,

(3) for every $a \in \mathcal{A} \backslash\{0\}$ there exists $t \in \mathbb{R} \backslash\{0\}$ such that $a-t$ is not invertible in $\mathcal{A}$.

Then there exists a compact Hausdorff space $\Omega$ such that $\mathcal{A}$ and $\mathcal{C}(\Omega, \mathbb{R})$ are isomorphic as real Banach algebras.

Proof. Let $\mathcal{A} \times \mathcal{A}$ be the complexification of the real Banach algebra $\mathcal{A}$. Then $\mathcal{A} \times \mathcal{A}$ becomes a commutative Banach algebra with unit. It is known that the mapping $\mathcal{A} \ni a \mapsto(a, 0) \in \mathcal{A} \times \mathcal{A}$ is a real algebra monomorphism which preserves units and invertibility of elements (i.e. if $(a, 0)$ is invertible in $\mathcal{A} \times \mathcal{A}$, then $a$ is invertible in $\mathcal{A}$ ) and it is a homeomorphism onto its range. Define a continuous involution on $\mathcal{A} \times \mathcal{A}$ by

$$
\mathcal{A} \times \mathcal{A} \ni(a, b) \mapsto(a, b)^{*}:=(a,-b) \in \mathcal{A} \times \mathcal{A} .
$$

We show that $\mathcal{A} \times \mathcal{A}$ satisfies condition (2) of Theorem 4.1. Let $\Omega$ denote the carrier space of $\mathcal{A} \times \mathcal{A}$ and $\Phi: \mathcal{A} \times \mathcal{A} \rightarrow \mathcal{C}(\Omega)$ be its Gelfand transform.

First we prove that the involution (4-1) is Hermitian. Take $(a, b) \in \mathcal{A} \times$ $\mathcal{A}$ such that $(a, b)^{*}=(a, b)$. Then clearly $b=0$. We have to show that $\sigma((a, 0)) \subset \mathbb{R}$. It suffices to prove that $(a, 0)-i$ is invertible in $\mathcal{A} \times \mathcal{A}$. By (1), $1+a^{2}$ is invertible in $\mathcal{A}$. Let $c \in \mathcal{A}$ be the inverse of $1+a^{2}$. Then

$$
((a, 0)-i) \cdot[((a, 0)+i) \cdot(c, 0)]=(a,-1) \cdot(a, 1) \cdot(c, 0)=\left(a^{2}+1,0\right) \cdot(c, 0)=(1,0) \text {. }
$$

Now we prove that the algebra $\mathcal{A} \times \mathcal{A}$ is semisimple. Suppose that $(a, b) \in$ $\mathcal{A} \times \mathcal{A}$ and $\sigma((a, b))=\{0\}$. By commutativity, we have $\sigma\left(\left(a^{2}+b^{2}, 0\right)\right)=$ $\sigma\left((a, b) \cdot(a, b)^{*}\right)=\{0\}$, so $\Phi((a, 0))^{2}+\Phi((b, 0))^{2}=\Phi\left(\left(a^{2}, 0\right)\right)+\Phi\left(\left(b^{2}, 0\right)\right)=$ $\Phi\left(\left(a^{2}+b^{2}, 0\right)\right)=0$. Since the involution is Hermitian and $(x, 0)^{*}=(x, 0)$ for $x \in \mathcal{A}$, the functions $\Phi((a, 0))$ and $\Phi((b, 0))$ are real-valued. But the sum of their squares equals 0 , so $\Phi((a, 0))=\Phi((b, 0))=0$. If $a \neq 0$, then, by (3), there exists a real $t \neq 0$ such that $a-t$ is not invertible in $\mathcal{A}$. Then $(a, 0)-t=(a-t, 0)$ is not invertible in $\mathcal{A} \times \mathcal{A}$ and consequently $t$ belongs to the range of $\Phi((a, 0))$, a contradiction. Therefore $a=0$ and similarly $b=0$.

It remains to prove that each $(a, b) \in \mathcal{A} \times \mathcal{A}$ with nonnegative spectrum has a square root with nonnegative spectrum. Since the involution is Hermitian and $\mathcal{A} \times \mathcal{A}$ is semisimple, $(a, b)^{*}=(a, b)$, which gives $b=0$. Thus 
$\sigma((a, 0)) \subset[0, \infty)$. This means that $a$ is nonnegative in $\mathcal{A}$. By $(2)$, there exists $c \in \mathcal{A}$ which is nonnegative in $\mathcal{A}$ and $c^{2}=a$. Then $\sigma((c, 0)) \subset[0, \infty)$ and $(c, 0)^{2}=\left(c^{2}, 0\right)=(a, 0)=(a, b)$, which completes the proof of our claim.

Finally, $\mathcal{A} \times \mathcal{A}$ satisfies condition (2) of Katznelson's theorem. Thus $\Phi$ is an isomorphism between $\mathcal{A} \times \mathcal{A}$ and $\mathcal{C}(\Omega)$. It is easy to check that $\left.\Phi(\mathcal{A} \times\{0\})=\mathcal{C}(\Omega, \mathbb{R}){ }^{13}\right)$. So the required isomorphism is $\mathcal{A} \ni a \mapsto$ $\Phi((a, 0)) \in \mathcal{C}(\Omega, \mathbb{R})$.

4.3. Lemma. Let $\mathcal{A}$ be a (*)-algebra such that $\operatorname{cl}[(*)-\operatorname{Alg}(A)] \subset \mathcal{A}$ for $A \in \mathcal{A}$. Then

$$
\sigma_{\mathcal{B}(\mathcal{H})}(A)=\sigma_{\mathcal{A}}(A), \quad A \in \mathcal{A}
$$

where $\sigma_{\mathcal{A}}(A)=\mathbb{C} \backslash\left\{\lambda \in \mathbb{C}: A-\lambda \in \mathcal{G}(\mathcal{H}),(A-\lambda)^{-1} \in \mathcal{A}\right\}(A \in \mathcal{A})$.

Proof. The inclusion " $\subset$ " is clear. Let $\mathcal{A}_{0}:=\operatorname{cl}[(*)-\operatorname{Alg}(A)]$. Then, by assumption, $\mathcal{A}_{0} \subset \mathcal{A}$. Therefore it suffices to prove that $\sigma_{\mathcal{A}_{0}}(A) \subset \sigma(A)$. Let $G \in \mathcal{G}(\mathcal{H})$ be any operator such that $N:=G^{-1} A G$ is normal. Then $\mathcal{A}_{0}=G \cdot \mathcal{C}^{*}(N) \cdot G^{-1}\left({ }^{14}\right)$. Now the conclusion follows from the well known equality $\sigma(N)=\sigma_{\mathcal{C}^{*}(N)}(N)$ (which is a consequence of the fact that each noninvertible element in $\mathcal{C}^{*}(N)$ is a topological divisor of zero).

Given a scalar operator $A$ we denote by $\mathcal{C}^{(*)}(A)$ the closure $($ in $\mathcal{B}(\mathcal{H})$ ) of (*)-Alg $(A)$. A simple argument (as in the proof of Lemma 4.3) shows that $\mathcal{C}^{(*)}(A)$ is a jointly scalar $\mathcal{C}^{(*)}$-algebra.

4.4. THEOREM. Every abelian $\mathcal{C}^{(*)}$-algebra is jointly scalar $\left({ }^{15}\right)$.

Proof. Let $\mathcal{A}$ be an abelian $\mathcal{C}^{(*)}$-algebra. We may assume that $I \in \mathcal{A}$ (because we can always take $\mathcal{A}+\mathbb{C} \cdot I$ which is a $\mathcal{C}^{(*)}$-algebra with unit). Then, by Lemma 4.3 , the spectrum (in $\mathcal{B}(\mathcal{H})$ ) of each element of $\mathcal{A}$ is equal to its spectrum with respect to $\mathcal{A}$. Hence $\mathcal{A}$ is a (commutative) semisimple algebra (Proposition 2.1) and $(*)$ is a Hermitian involution on $\mathcal{A}$ (Corollary 3.5$)$.

If $A \in \mathcal{A}$ has a nonnegative spectrum, then there exists $B \in \mathcal{C}^{(*)}(A)$, also with nonnegative spectrum, such that $B^{2}=A$ (because $A$ is similar to nonnegative operator). Since $\mathcal{A}$ is closed, we conclude that $\mathcal{C}^{(*)}(A) \subset \mathcal{A}$ and hence $B \in \mathcal{A}$. This means that each element of $\mathcal{A}$ with nonnegative spectrum has a square root with nonnegative spectrum. By Theorem 4.1, there exists a compact Hausdorff space $\Omega$ and an algebra isomorphism $\Phi: \mathcal{C}(\Omega) \rightarrow \mathcal{A}$. Hence Theorem 1.13(i) completes the proof.

$\left({ }^{13}\right)$ Since the algebra is semisimple and the involution is Hermitian, $\Phi$ preserves the involution.

$\left({ }^{14}\right)$ Here $\mathcal{C}^{*}(N)=\mathcal{C}^{*}(\{N, I\})$.

$\left({ }^{15}\right)$ The assumption that the algebra is abelian is not restrictive since any algebra of normal operators is commutative; we do not know if this hypothesis can be omitted (see Example 4.10). 
4.5. Corollary. (1) The operation of taking quasi-adjoints is continuous on any abelian $\mathcal{C}^{(*)}$-algebra $\left.{ }^{(16}\right)$.

(2) If $\mathcal{A}_{0}$ is an abelian $\mathcal{C}^{(*)}$-algebra, then $\mathcal{A}:=\left\{A \in \mathcal{A}_{0}: A=A^{(*)}\right\}$ is a closed abelian real algebra of operators similar to selfadjoint ones and $\mathcal{A}_{0}=\mathcal{A}+i \mathcal{A}$.

Proof. By Theorem 4.4, the operation of taking quasi-adjoints on abelian $\mathcal{C}^{(*)}$-algebras is similar to the operation of taking standard adjoints, which is continuous. This yields (1) and (2).

According to Corollary 4.5, if the closure of a $(*)$-algebra is a $(*)$-algebra, then taking quasi-adjoints is continuous on it. But this property may not be true in general (see Remark 4.11).

We now state a generalization of Theorem 4.4.

4.6. TheOREm. Let $\mathcal{A} \subset \mathcal{B}(\mathcal{H})$ be an abelian real algebra of operators similar to selfadjoint ones. If $\mathcal{A}$ is closed, then $\mathcal{A}$ is jointly scalar.

Proof. We may assume that $I \in \mathcal{A}($ consider $\mathcal{A}+\mathbb{R} \cdot I$ instead of $\mathcal{A})$. So $\mathcal{A}$ is an abelian real Banach algebra with unit. It is easy to check (arguing as in the proof of Theorem 4.4) that $\mathcal{A}$ satisfies the assumptions of Theorem 4.2: it is invariant under taking inverses (i.e. if $A \in \mathcal{A}$ is invertible in $\mathcal{B}(\mathcal{H})$, then $A^{-1}$ belongs to $\mathcal{A}$ ); also the square root of any nonnegative element in $\mathcal{A}$ belongs to $\mathcal{A}$ (we can approximate the square root by polynomials with real coefficients). Hence $\mathcal{A}$ is isomorphic (and homeomorphic) to the algebra $\mathcal{C}(\Omega, \mathbb{R})$, where $\Omega$ is a compact Hausdorff space. By Proposition 1.12 we can extend the isomorphism from $\mathcal{C}(\Omega, \mathbb{R})$ onto $\mathcal{A}$ to a continuous algebra homomorphism between $\mathcal{C}(\Omega)$ and $\mathcal{B}(\mathcal{H})$. Hence Theorem 1.13 completes the proof.

4.7. Corollary. Let $\mathcal{A}$ be an abelian (*)-algebra. Then

(1) $\mathcal{A}_{C C}$ is jointly scalar provided $\mathcal{A}$ is closed,

(2) there exists a maximal (with respect to inclusion) commutative $\mathcal{C}^{(*)}$ algebra $\mathcal{A}_{0}$ with unit such that $\mathcal{A} \subset \mathcal{A}_{0}$ provided $\mathcal{A}$ is closed,

(3) $\mathcal{A}$ is a maximal abelian $\mathcal{C}^{(*)}$-algebra if and only if $\mathcal{A}=\mathcal{A}_{C}$,

(4) $\mathcal{A}$ is jointly scalar if and only if $\operatorname{cl} \mathcal{A}$ is a separately scalar set and the operation of taking quasi-adjoints is continuous on $\mathcal{A}$.

Proof. (1) follows from Theorems 4.4 and 3.4.

(2) If $\mathcal{A}$ is closed, then, by Theorem 4.4, there exists $G \in \mathcal{G}(\mathcal{H})$ such that $G^{-1} A G$ is normal for all $A \in \mathcal{A}$. Then $\mathcal{F}:=\left\{G^{-1} A G: A \in \mathcal{A}\right\}$ is a commutative $\mathcal{C}^{*}$-algebra and there exists a $\mathcal{C}^{*}$-algebra $\mathcal{F}_{0}$ such that $\mathcal{F} \subset \mathcal{F}_{0}$ and $\mathcal{F}_{0}=\left(\mathcal{F}_{0}\right)_{C}$. Put $\mathcal{A}_{0}:=\left\{G N G^{-1}: N \in \mathcal{F}_{0}\right\}$. Then $\mathcal{A}_{0}$ is a $\mathcal{C}^{(*)}$-algebra

$\left({ }^{16}\right)$ There is a much stronger theorem which states that involutions on semisimple (not necessarily commutative) Banach algebras are continuous (see [B-D, Theorem V.36.2]). 
with unit such that $\mathcal{A} \subset \mathcal{A}_{0}$ and $\mathcal{A}_{0}=\left(\mathcal{A}_{0}\right)_{C}$. Now it is easy to show that $\mathcal{A}_{0}$ is maximal.

(3) By the proof of (2), we see that any abelian $\mathcal{C}^{(*)}$-algebra is contained in some abelian $\mathcal{C}^{(*)}$-algebra which coincides with its commutant. This proves the "only if" part of (3). The other implication is clear.

(4) The "only if" implication follows from Theorem 3.4.

To prove the converse, take $\mathcal{A}_{0}:=\operatorname{cl} \mathcal{A}$. Then $\mathcal{A}_{0}$ is an abelian closed algebra which, by assumption, is separately scalar. By Theorem 4.4, it is enough to prove that $\mathcal{A}_{0}$ is invariant under taking quasi-adjoints. If $A \in \mathcal{A}_{0}$, then there exists a sequence $\left(A_{n}\right)_{n=1}^{\infty} \subset \mathcal{A}$ such that $\lim _{n \rightarrow \infty} A_{n}=A$. Since taking quasi-adjoints is continuous (and $\mathbb{R}$-linear) on $\mathcal{A}$, there exists $B \in \mathcal{A}_{0}$ such that $\lim _{n \rightarrow \infty} A_{n}^{(*)}=B$. Then

$$
\begin{array}{ll}
\frac{A_{n}+A_{n}^{(*)}}{2} \rightarrow \frac{A+B}{2} & (n \rightarrow \infty), \\
\frac{A_{n}-A_{n}^{(*)}}{2 i} \rightarrow \frac{A-B}{2 i} & (n \rightarrow \infty) .
\end{array}
$$

In each of these two relations, the left-hand side operators commute and are similar to selfadjoint operators, so their spectra are subsets of the real line; hence $\left({ }^{17}\right) \sigma\left(\frac{A+B}{2}\right) \subset \mathbb{R}$ and $\sigma\left(\frac{A-B}{2 i}\right) \subset \mathbb{R}$. But both $\frac{A+B}{2}$ and $\frac{A-B}{2 i}$ are scalar and therefore similar to selfadjoint operators (because their spectra are real). This means that

$$
\left(\frac{A+B}{2}\right)^{(*)}=\frac{A+B}{2} \text { and }\left(\frac{A-B}{2 i}\right)^{(*)}=\frac{A-B}{2 i} \text {. }
$$

Finally, we have (recall that the operators commute)

$$
\begin{aligned}
A^{(*)} & =\left(\frac{A+B}{2}+i \frac{A-B}{2 i}\right)^{(*)}=\left(\frac{A+B}{2}\right)^{(*)}-i\left(\frac{A-B}{2 i}\right)^{(*)} \\
& =\frac{A+B}{2}-i \cdot \frac{A-B}{2 i}=B \in \mathcal{A}_{0} .
\end{aligned}
$$

Therefore $\mathcal{A}_{0}$ is a closed (*)-algebra, so we can apply Theorem 4.4.

We now state the converse to part (2) of Corollary 4.5.

4.8. Corollary. Let $\mathcal{A} \subset \mathcal{B}(\mathcal{H})$ be an abelian real algebra of operators similar to selfadjoint ones. If $\mathcal{A}$ is closed, then $\mathcal{A}_{0}:=\mathcal{A}+i \mathcal{A}$ is an abelian $\mathcal{C}^{(*)}$-algebra ${ }^{18}$ ) such that $\mathcal{A}=\left\{A \in \mathcal{A}_{0}: A=A^{(*)}\right\}$.

$\left({ }^{17}\right)$ Here it is important that the operators commute (then the spectrum of the limit is the limit of the spectra in the Hausdorff distance on the space of all nonempty compact subsets of the complex plane; this property follows from [B-D, Propositions I.5.17 and I.5.18]).

$\left({ }^{18}\right)$ In particular, it is a closed set. 
Proof. By Theorem 4.6, $\mathcal{A}$ is similar to some closed abelian (real) algebra of selfadjoint operators, say $\mathcal{A}^{\prime}$. Then $\mathcal{A}^{\prime}+i \mathcal{A}^{\prime}$ is a $\mathcal{C}^{*}$-algebra such that $\mathcal{A}^{\prime}=\left\{A \in \mathcal{A}^{\prime}+i \mathcal{A}^{\prime}: A=A^{*}\right\}$. This completes the proof.

The following fact can be deduced from Theorems 1.13 and 4.4.

4.9. Corollary. Let $\mathcal{A} \subset \mathcal{B}(\mathcal{H})$ be an abelian algebra with unit. Then the following conditions are equivalent:

(1) $\mathcal{A}$ is a $\mathcal{C}^{(*)}$-algebra,

(2) $\mathcal{A}$ is closed and the Gelfand transform of $\mathcal{A}$ is an algebra isomorphism between $\mathcal{A}$ and the algebra of all complex-valued continuous functions on its carrier space,

(3) there exists a (nonempty) compact Hausdorff space $\Omega$ and a continuous algebra homomorphism $\Phi: \mathcal{C}(\Omega) \rightarrow \mathcal{B}(\mathcal{H})$ preserving units such that $\Phi(\mathcal{C}(\Omega))=\mathcal{A}$.

We conclude the paper with two examples.

4.10. EXAmpLes. (I) For normal operators $M, N$ the following implication holds true: if $M+N$ and $M+i N$ are normal, then $M$ and $N$ commute. This is no longer true for scalar operators as the following example shows. Let $\mathcal{H}:=\mathbb{C}^{2}, P:=\left(\begin{array}{ll}1 & 1 \\ 0 & 0\end{array}\right)$ and $Q:=\left(\begin{array}{ll}1 & 0 \\ 0 & 0\end{array}\right)$. It is easy to check that $P^{2}=P, Q^{2}=Q, P Q \neq Q P$ and, by Proposition $2.9, P+\lambda Q$ is scalar for $\lambda \in \mathbb{C} \backslash\{-1\}$.

(II) As the following example shows, the operation of taking quasiadjoints is not additive in general, where the additivity would mean that if $A, B, A+B$ are scalar, then $(A+B)^{(*)}=A^{(*)}+B^{(*)}$. Let $\mathcal{H}:=\mathbb{C}^{2}$, $P:=\left(\begin{array}{ll}1 & 0 \\ 1 & 0\end{array}\right)$ and $Q:=\left(\begin{array}{cc}1 & -2 \\ 0 & 0\end{array}\right)$. It is easy to check that $P^{2}=P, Q^{2}=Q$ (so $\left.P^{(*)}=P, Q^{(*)}=Q\right)$ and $P+Q$ is scalar but not similar to any selfadjoint operator (because $\sigma(P+Q) \not \subset \mathbb{R}$ ).

4.11. REMARK. Notice that

(a) the operation of taking quasi-adjoints is not continuous on abelian (*)-algebras in general,

(b) the norm limit of a sequence of commuting scalar operators is not a scalar operator in general.

Proof. First we construct an auxiliary sequence of scalar operators. Let $\mathcal{H}:=\mathbb{C}^{3}$. Set

$$
U:=\left(\begin{array}{ccc}
0 & 0 & 1 \\
1 & 0 & 0 \\
0 & 1 & 0
\end{array}\right), \quad A_{n}:=\left(\begin{array}{ccc}
1 & 0 & 0 \\
0 & 1 / n & 0 \\
0 & 0 & n
\end{array}\right), \quad n \geq 1 .
$$

One can check that $U$ is unitary and each $A_{n}$ belongs to $\mathcal{G}_{+}(\mathcal{H})$. Therefore 
each $U_{n}:=A_{n}^{-1} U A_{n}(n \geq 1)$ is scalar. It is clear that

$$
U_{n}=\left(\begin{array}{ccc}
0 & 0 & n \\
n & 0 & 0 \\
0 & 1 / n^{2} & 0
\end{array}\right), \quad U_{n}^{(*)}=U_{n}^{-1}=\left(\begin{array}{ccc}
0 & 1 / n & 0 \\
0 & 0 & n^{2} \\
1 / n & 0 & 0
\end{array}\right), \quad n \geq 1
$$

Moreover, $\left\|U_{n}\right\|=n$ and $\left\|U_{n}^{(*)}\right\|=n^{2}(n \geq 1)\left(^{19}\right)$. Finally, we define $V_{n}:=\left(1 / n^{2}\right) U_{n}(n \geq 1)$. Then $\left(V_{n}\right)_{n=1}^{\infty}$ is a sequence of scalar operators such that $\lim _{n \rightarrow \infty}\left\|V_{n}\right\|=0$ though $\left\|V_{n}^{(*)}\right\|=1(n \geq 1)$.

We can now prove both parts of the Remark. Clearly, neither part can be realized in a finite-dimensional Hilbert space. Let $\mathcal{H}$ be any infinitedimensional complex Hilbert space.

(a) There exists a sequence $\left\{\mathcal{H}_{n}\right\}_{n=0}^{\infty}$ of closed linear subspaces of $\mathcal{H}$ such that $\operatorname{dim} \mathcal{H}_{n}=3$ for $n \geq 1$ and

$$
\mathcal{H}=\bigoplus_{n=0}^{\infty} \mathcal{H}_{n}
$$

We can identify $\mathcal{H}_{n}$ with $\mathbb{C}^{3}$ and consider $V_{n}$, defined above, as an operator on $\mathcal{H}_{n}(n \geq 1)$. Then we define a sequence $\left(M_{n}\right)_{n=1}^{\infty} \subset \mathcal{B}(\mathcal{H})$ by

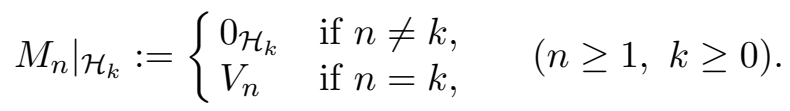

It is easy to check that this is a sequence of scalar operators which commute and $\lim _{n \rightarrow \infty}\left\|M_{n}\right\|=\lim _{n \rightarrow \infty}\left\|V_{n}\right\|=0$ though $\left\|M_{n}^{(*)}\right\|=\left\|V_{n}^{(*)}\right\|=1 \quad(n \geq 1)$. This means that $(*)$ is not continuous in general (by Corollary 3.5).

(b) Suppose, contrary to our claim, that the norm limit of any sequence of commuting scalar operators is always scalar. We show that $(*)$ is then continuous on any abelian (*)-algebra, which is impossible (by (a)).

Let $\mathcal{A}_{0}$ be any abelian (*)-algebra. By the Kuratowski-Zorn lemma there exists a maximal abelian (*)-algebra $\mathcal{A}$ with unit containing $\mathcal{A}_{0}$.

Let $A \in \operatorname{cl}_{\mathcal{B}(\mathcal{H})} \mathcal{A}$. By our assumptions, $A$ is scalar (because $\mathcal{A}$ is abelian). It is easy to check that $A \in \mathcal{A}_{C}$, so the set $\mathcal{A} \cup\{A\}$ is abelian and separately scalar and therefore there exists an abelian $(*)$-algebra $\mathcal{A}_{1}$ with unit such that $\mathcal{A} \cup\{A\} \subset \mathcal{A}_{1}$ (by Corollary 3.5). Since $\mathcal{A}$ is maximal, we see that $\mathcal{A}=\mathcal{A}_{1}$ and in consequence $A \in \mathcal{A}$. This means that $\mathcal{A}$ is closed, i.e. it is a commutative $\mathcal{C}^{(*)}$-algebra. Hence, by Corollary 4.5 , taking quasi-adjoints is continuous on $\mathcal{A}$ and hence on $\mathcal{A}_{0}$. But this contradicts (a).

The author would like to thank Prof. Jan Stochel for helpful information and advice.

$$
\left.{ }^{19}\right)\left\|\left(\begin{array}{lll}
0 & 0 & t \\
u & 0 & 0 \\
0 & v & 0
\end{array}\right)\right\|=\max \{|t|,|u|,|v|\}(t, u, v \in \mathbb{C}) .
$$




\section{References}

[Bab] K. I. Babenko, On conjugate functions, Dokl. Akad. Nauk SSSR 62 (1948), 157160 (in Russian).

[B-N] N. E. Benamara and N. Nikolski, Resolvent tests for similarity to a normal operator, Proc. London Math. Soc. 78 (1999), 585-626.

[B-D] F. F. Bonsall and N. J. Duncan, Complete Normed Algebras, Springer, Berlin, 1973.

[Ca1] J. A. van Casteren, A problem of Sz.-Nagy, Acta Sci. Math. (Szeged) 42 (1980), 189-194.

[Ca2] - Operators similar to unitary or selfadjoint ones, Pacific J. Math. 104 (1983), 241-255.

[Day] M. M. Day, Normed Linear Spaces, Springer, Berlin, 1958.

[DS1] N. Dunford and J. T. Schwartz, Linear Operators, Part I, Interscience Publ., New York, 1958.

[DS3] —, - Linear Operators, Part III, Wiley-Interscience, New York, 1971.

[G-K] I. Ts. Gokhberg and M. G. Kreŭn, On a description of contraction operators similar to unitary ones, Funktsional. Anal. i Prilozhen. 1 (1967), no. 1, 38-60 (in Russian).

[Kat] Y. Katznelson, Sur les algèbres dont les éléments non négatifs admettent des racines carrées, Ann. Sci. École Norm. Sup. (3) 77 (1960), 167-174.

[Lor] E. R. Lorch, Bicontinuous linear transformations in certain vector spaces, Bull. Amer. Math. Soc. 45 (1939), 564-569.

[Nab] S. N. Naboko, Conditions for similarity to unitary and selfadjoint operators, Funktsional. Anal. i Prilozhen. 18 (1984), no. 1, 16-27 (in Russian).

[SzN] B. Sz.-Nagy, On uniformly bounded linear transformations in Hilbert space, Acta Sci. Math. (Szeged) 11 (1947), 152-157.

Institute of Mathematics

Jagiellonian University

Reymonta 4

30-059 Kraków, Poland

E-mail: Piotr.Niemiec@im.uj.edu.pl

Received September 29, 2000

Revised version March 19, 2001 